\title{
$\mathrm{UNLV}||_{\text {LIBRARIES }}^{\mathrm{UNIVRSTY}}$
}

Mechanical Engineering Faculty Publications

Mechanical Engineering

8-15-2005

\section{Adaptive Control of a Projectile Fin Using Piezoelectric Elastic Beam}

Smitha Mani
University of Nevada, Las Vegas

Sahjendra N. Singh

University of Nevada, Las Vegas, sahjendra.singh@unlv.edu

Surya Kiran Parimi

University of Nevada, Las Vegas

Woosoon Yim

University of Nevada, Las Vegas, woosoon.yim@unlv.edu

Mohamed B. Trabia

University of Nevada, Las Vegas, mbt@me.unlv.edu

Follow this and additional works at: https://digitalscholarship.unlv.edu/me_fac_articles

Part of the Aerodynamics and Fluid Mechanics Commons, Electronic Devices and Semiconductor

Manufacturing Commons, Energy Systems Commons, and the Structures and Materials Commons

\section{Repository Citation}

Mani, S., Singh, S. N., Parimi, S. K., Yim, W., Trabia, M. B. (2005). Adaptive Control of a Projectile Fin Using Piezoelectric Elastic Beam. AIAA Guidance, Navigation, and Control Conference 2005, 3 2247-2257. AIAA. https://digitalscholarship.unlv.edu/me_fac_articles/533

This Conference Proceeding is protected by copyright and/or related rights. It has been brought to you by Digital Scholarship@UNLV with permission from the rights-holder(s). You are free to use this Conference Proceeding in any way that is permitted by the copyright and related rights legislation that applies to your use. For other uses you need to obtain permission from the rights-holder(s) directly, unless additional rights are indicated by a Creative Commons license in the record and/or on the work itself.

This Conference Proceeding has been accepted for inclusion in Mechanical Engineering Faculty Publications by an authorized administrator of Digital Scholarship@UNLV. For more information, please contact

digitalscholarship@unlv.edu. 


\title{
Adaptive Control of a Projectile Fin Using Piezoelectric Elastic Beam
}

\author{
Smitha Mani* and Sahjendra N. Singh ${ }^{\dagger}$ \\ Surya K. Parimi ${ }^{\ddagger}$, Woosoon Yim $^{\S}$ and Mohamed Trabia \\ University of Nevada, Las Vegas, NV-89154-4026
}

\begin{abstract}
This paper treats the question of model reference adaptive control of a projectile fin using a piezoelectric actuator. The hollow projectile fin is rigid, within which a flexible cantilever beam with a piezoelectric active layer is mounted. The model of the fin-beam system includes the aerodynamic moment which is a function of angle of attack of the projectile. The rotation angle of the fin is controlled by deforming the flexible beam which is hinged at the tip of the rigid fin. Based on the command generator tracker concept, a model reference adaptive fin angle controller is designed. For the design of the control law, a linear combination of the fin angle and fin's angular rate is chosen as the controlled output variable. In the closed-loop system, the controlled output variable tracks the reference trajectory and the fin angle asymptotically converges to the desired value and the elastic modes converges to their equilibrium values. Simulation results are presented which show that in the closed-loop system, the fin angle is precisely controlled in spite of uncertainties in the fin-beam parameters and the aerodynamic moment coefficients
\end{abstract}

\section{Introduction}

The use of surface-mounted or bonded piezoelectric actuators for the shape control of intelligent structure has gained widespread acceptance recently. Applications can be found in many areas including the shape control of metallic or composite plates or beams ${ }^{1,2}$. Applications also involved actuation of various types of aircraft structural members such as wings, fins, or rotor blade ${ }^{3-6}$. Advantages of this approach are mainly due to the integration of the actuators into the structural members itself, thus saving the space required for servo motors, force transmission devices, or hydraulic systems ${ }^{4}$. This advantage becomes even more important when small aerial vehicles such as unmanned aircraft, small missiles, guided munitions, and projectiles, are examined. Piezoelectric twist actuators used for this application are based on anisotropic straining of the host structure using directionally attached isotropic actuator ${ }^{5}$ or using piezoelectric fibers integrated into the composite structural members ${ }^{6}$. General formulation and solution procedures for an analytical model for a composite laminated plate with isotropic or anisotropic active layers is derived in Refs. 3,4. The design of active controllers using piezoelectric actuators for vibration, force and position control of systems have been considered by various authors ${ }^{7-12}$.

Traditionally, for the path control of missiles and projectiles, maneuvering forces and moments are generated by fin angle control using mechanical actuators which are bulky and slow. For high performance projectiles, there is a need to develop more efficient actuation mechanisms. Recently, the development of a smart fin (fin-beam model) has been considered ${ }^{12,13}$. This fin has an outer hollow rigid body inside which resides a hinged flexible beam with a piezoelectric active layer. The control of the fin angle is then accomplished by deforming the beam. The design of the controller of

\footnotetext{
*Graduate Student, Department of Electrical \& Computer Engineering.

${ }^{\dagger}$ Professor, Department of Electrical \& Computer Engineering

$\ddagger$ Graduate Student, Department of Mechanical Engineering.

$\S$ Professor, Department of Mechanical Engineering

I Professor, Department of Mechanical Engineering
} 
Ref. 13 is based on a modeling error compensation approach in which the lumped uncertainties are estimated using a high-gain observer. This requires precise measurement of the fin angle for stability in the closed-loop system. A fuzzy controller has been designed in Ref. 14 for the control of this fin. Of course, for the fuzzy controller design, the designer first has to develop a number of if-then rules which often are not easy to obtain. Ref. 23 provides an adaptive controller, based on inverse feedback linearization technique. However, this requires synthesis using either the state feedback or discontinuous output feedback control.

Flexible structures are essentially infinite dimensional systems; however often finite dimensional models by neglecting the higher modes are used for analysis and design. The models of flexible structures are generally obtained by solving the eigenvalue problem resulting from finite element methods. However, it is well known that the resulting fidelity of model parameters degrades drastically for higher modes. Researchers have made considerable effort to design controllers for the control of flexible structures. Ref. 15 provides a good review of literature in which readers will find several references. For flexible structures, controller designs based on feedback linearization, passivity concepts and adaptive techniques have been attempted ${ }^{16-21}$. But the methods based on passivity ${ }^{17}$ have advantage over other design techniques since the passivity approach does not rely on model truncation or higher-order models of the structure and is independent of the numerical values of the model data.

The contribution of the paper lies in the design of an adaptive control system for the control of a projectile fin. The projectile fin which is hollow but rigid, is controlled by deforming a cantilever flexible beam, which is mounted inside the fin. The model chosen here is similar to that reported in Ref. 13. A finite dimensional model is used for study, but the design is independent of the truncation order of the model obtained using finite element method. The model includes the aerodynamic moment affecting the fin motion which is a function of the angle of attack of the projectile. For the trajectory control of the fin angle, a judicious choice of a controlled output variable which is a linear combination of the tip position and velocity of the flexible beam is made. Based on the command generator tracker concept, a model reference adaptive control law is designed. In the closed-loop system the fin angle asymptotically converges to the target fin angle generated by a command generator. In the closed-loop all the elastic modes converge to their equilibrium values. Simulation results are presented which show that the designed adaptive control system accomplishes precise fin angle control in spite of uncertainties in the fin-beam parameters and the aerodynamic moment coefficients.

The organization of the paper is as follows. The mathematical model is presented in Section II. Section III presents the control law and simulation results are given in Section IV.

\section{Dynamic Model}

The model of the fin-beam system is shown in Figure 1. The flexible beam with a piezoelectric active layer bonded on the top surface, is hinged at one end to the fin and the other end is attached rigidly to the projectile body. The fin is free to rotate about an axis fixed to the projectile body. When the control voltage $u(x, t)$ is applied to the actuator, the induced strain in the actuator generates the bending moment $m$ that is expressed ${ }^{8}$ as

$$
m=c u(x, t)
$$

The constant $c$ can be obtained by considering geometrical and material properties of the beam and piezoelectric actuator. Considering the cross sectional geometry and force equilibrium along the axial direction, the constant $c$ can be expressed as ${ }^{9}$

$$
c=-d_{31} \frac{h_{p}+h_{b}}{2} \frac{E_{p} h_{b} E_{b}}{E_{p} h_{p}+E_{b} h_{b}} b
$$

where $d_{31}$ is the piezoelectric strain constant and $E_{p}$ and $E_{b}$ are Young's modulus of the piezoelectric actuator and the beam respectively. Other geometric parameters are shown in Fig. 1.

As shown in Fig. 1, an airfoil is connected to the beam actuator using a hinge. The airfoil is assumed to be rigid and its rotation is assumed to be small and planar. A finite element approach is used to describe the dynamics of the flexible beam, which is considered as composed of finite elements 
satisfying Euler-Bernoullis theorem. The beam is divided into $n$ elements with equal length of $L_{i}$. The displacement $w$ of any point on the beam element $i$ is described in terms of nodal displacement, $w_{i}$, and slope, $\phi_{i}$, at node $i$ and $i+1$, respectively and is expressed as

$$
w_{i}=N q_{i}
$$

where $q_{i}=\left(w_{i}, \phi_{i}, w_{i+1}, \phi_{i+1}\right)^{T}$ and $N=\left(N_{1}, N_{2}, N_{3}, N_{4}\right)$ is the shape function vector with

$$
\begin{aligned}
N_{1} & =\frac{1}{L_{i}^{3}}\left(2 x_{i}^{3}-3 x_{i}^{2} L_{i}+L_{i}^{3}\right) \\
N_{2} & =\frac{1}{L_{i}^{3}}\left(x_{i}^{3} L_{i}-2 x_{i}^{2} L_{i}^{2}+x_{i} L_{i}^{3}\right) \\
N_{3} & =\frac{1}{L_{i}^{3}}\left(-2 x_{i}^{3}+3 x_{i}^{2} L_{i}\right) \\
N_{4} & =\frac{1}{L_{i}^{3}}\left(x_{i}^{3} L_{i}-x_{i}^{2} L_{i}^{2}\right)
\end{aligned}
$$

where $x_{i}$ is the element local coordinate variable defined along the beam neutral axis. The kinetic energy of an element $i$ becomes

$$
T_{i}=\int_{0}^{L_{i}} \rho_{i} \dot{w}^{T} \dot{w} d x_{i} \doteq \frac{1}{2} \dot{q}_{i}^{T} M_{i} \dot{q}_{i}
$$

where $M_{i}\left(=\int_{0}^{L_{i}} \rho_{i} N^{T} N d x_{i}\right)$ is a mass matrix and $\rho_{i}$ is a combined density of the beam and piezoelectric actuator per unit length.

The potential energy of an element $i$ is

$$
V_{i}=\frac{1}{2} \int_{0}^{L_{i}} \frac{1}{E_{i} I_{i}}\left(E_{i} I_{i} \frac{\partial^{2} w}{\partial x_{i}^{2}}+c u\right)^{T}\left(E_{i} I_{i} \frac{\partial^{2} w}{\partial x_{i}^{2}}+c u\right) d x_{i}
$$

where $E_{i} I_{i}$ is the product of Youngs modulus of elasticity by the cross-sectional area moment of inertia for the equivalent beam for an element $i$. If the piezoelectric actuator has a uniform geometry and that a uniform voltage is applied along its length, $u$ can be assumed to be function of time only. The potential energy of an element can be further expressed as,

$$
V_{i}=\frac{1}{2} q_{i} K_{i} q_{i}+q_{i}\left(\int_{0}^{L_{i}} \frac{\partial^{2} N}{\partial x_{i}^{2}} d x_{i}\right) c u(t)+\frac{1}{2 E_{i} I_{i}} c^{2} u^{2}(t)
$$

where the stiffness matrix $K_{i}$ becomes

$$
K_{i}=\int_{0}^{L_{i}} E_{i} I_{i}\left(\frac{\partial^{2} N}{\partial x_{i}^{2}}\right)^{T}\left(\frac{\partial^{2} N}{\partial x_{i}^{2}}\right) d x_{i}
$$

The kinetic energy of the rigid fin is

$$
T_{f}=\frac{1}{2} \dot{w}_{n+1}^{T} \frac{J_{f}}{L^{2}} \dot{w}_{n+1}
$$

where $J_{f}$ is the mass moment of inertia of the fin.

Using the Lagrangian dynamics, the equations of motion for an element $i$ becomes

$$
M_{i} \ddot{q}_{i}+K q_{i}=B_{i}(-c u(t)), \quad i=1, \ldots, n-1
$$

where $B_{i}=(0,-1,0,-1)^{T}$ which represent two concentrated moments at two nodes of the element $i$. For the last element or $i=n$, the equation of motion including the mass of the rigid fin becomes

$$
M_{n} \ddot{q}_{n}+\left[\begin{array}{cccc}
0 & 0 & 0 & 0 \\
0 & 0 & 0 & 0 \\
0 & 0 & \frac{J_{f}}{L^{2}} & 0 \\
0 & 0 & 0 & 0
\end{array}\right] \ddot{q}_{n}+K q_{n}=B_{n}(-c u(t))
$$


The equations derived for each element can be assembled after expansion and matrix reduction from the boundary conditions of the cantilever beam as follows,

$$
M \ddot{q}+K q=B_{0} u(t)
$$

where $q=\left(w_{2}, \phi_{2}, \ldots, w_{n+1}, \phi_{n+1}\right)^{T} \in \Re^{2 n}$. Considering the hinge connection between the beam actuator and the blade, the fin angle can be expressed as

$$
\theta=\tan ^{-1}\left(\frac{\delta_{t}}{L}\right)
$$

where $L$ is the total length of the beam and $\delta_{t}$ is the tip displacement of the beam. For small fin angle, it can be approximated as $\theta=\delta_{t} / L$.

The aerodynamic moment acting on the fin is a complicated function of the angle of attack of the projectile and the fin rotation angle. The data generated by the computational fluid dynamics show that the aerodynamic moment can be accurately modeled as a linear function of the fin angle and a reasonable model can be expressed as

$$
m_{a}=m_{a 0}(\alpha)+p_{a}(\alpha) \theta=m_{a 0}(\alpha)+p_{a}(\alpha) L^{-1} e^{\star T} q
$$

where $p_{a}(\alpha)$ is a polynomial in the angle of attack, $\alpha, p_{a}(\alpha)=p_{0}+p_{1} \alpha+\ldots . .+p_{k} \alpha^{k}$ ( $k$ is a positive integer) and $e^{\star T} \in \Re^{2 n}$ is a unit vector whose $(2 n-1)^{t h}$ element is one and rest are zero.

The modified fin-beam model including the aerodynamic force takes the form

$$
M \ddot{q}+K q=B_{0} u(t)+B_{a} m_{a}
$$

where $B_{a}=L^{-1}[0, \ldots 0,1,0]^{T} \in \Re^{2 n}$. Solving (15) gives

$$
\ddot{q}=-M^{-1} K_{m} q+M^{-1} B_{0} u(t)+M^{-1} B_{a} m_{a 0}(\alpha)
$$

where $K_{m}=K-p_{a}(\alpha) L^{-1} B_{a} e^{\star T}$.

The eigenvalues of $M^{-1} K_{m}$ are distinct positive real numbers. As such there exists a similarity transformation matrix $V$ formed by the eigenvectors of the matrix $M^{-1} K_{m}$ such that

$$
V^{-1} M^{-1} K_{m} V=\Omega^{2}
$$

where $\Omega^{2}=\operatorname{diag}\left(\Omega_{i}^{2}\right), i=1, \ldots, 2 n ; \Omega_{i} \neq \Omega_{j}, i \neq j$.

Defining $\eta=V^{-1} q$, one obtains from (16)

$$
\begin{gathered}
\ddot{\eta}=-\Omega^{2} \eta+V^{-1} M^{-1} B_{0} u(t)+V^{-1} M^{-1} B_{a} m_{a 0}(\alpha) \\
=-\Omega^{2} \eta+B_{1} u(t)+d
\end{gathered}
$$

where $B_{1}=V^{-1} M^{-1} B_{0} \in \Re^{2 n}$ and $d=V^{-1} M^{-1} B_{a} m_{a 0}(\alpha)$. The modal form (15) has no damping. However, there is nonzero structural damping for any elastic body. As such it is common to introduce a dissipation term proportional to the rate $\dot{\eta}$. Introducing a damping term of the form $2 D \Omega$, where $D=\operatorname{diag}\left(\zeta_{i}\right), i=1, \ldots, 2 n, \zeta_{i}>0$, one obtains the system

$$
\ddot{\eta}=-2 D \Omega \eta-\Omega^{2} \eta+B_{1} u
$$

The fin angle in new coordinate becomes

$$
\theta=L^{-1} e^{\star T} q=L^{-1} e^{\star T} V \eta=C_{0} \eta
$$

It is assumed that the system matrices $D, \Omega, B_{1}$ and $C_{0}$ are unknown. Furthermore, it is assumed that only the fin angle and the angular rate is measurable. Suppose that reference model of the form

$$
\begin{gathered}
\dot{x_{m}}=A_{m} x_{m}+B_{m} u_{m} \\
y_{m}=C_{m} x_{m}
\end{gathered}
$$

is given. We are interested in designing an adaptive control system such that the fin angle asymptotically tracks the reference trajectory $y_{m}$. Moreover, for synthesis only the measured angles $\theta$ and $\dot{\theta}$ are to be used. 


\section{Adaptive Control Law}

In the following derivation, adaptive controller will be designed assuming that the disturbance input $d$ is zero. Defining the state vector $x=\left(\eta^{T}, \dot{\eta}^{T}\right)^{T}$, a state variable representation of $(20)$ with $d=0$ takes the form

$$
\begin{gathered}
\dot{x}=\left[\begin{array}{cc}
0_{2 n \times 2 n} & I_{2 n \times 2 n} \\
-\Omega^{2} & -2 D \Omega
\end{array}\right] x+\left[\begin{array}{c}
0_{2 n \times 1} \\
B_{1}
\end{array}\right] u \\
\triangleq A x+B u
\end{gathered}
$$

We associate with the system (22), an output variable

$$
y=C x
$$

where $C$ is yet to be chosen.

For the design of controller based on CGT method ${ }^{16,17}$, almost strictly positive real (ASPR) condition for the system (19) and (20) is required ${ }^{25}$.

Definition: A system $\{A, B, C\}$ is ASPR if there exists a gain $K_{e}$ and symmetric positive definite matrices $P, Q \in \Re^{4 n \times 4 n}$ such that

$$
\begin{gathered}
P\left(A-K_{e} B C\right)+\left(A-K_{e} B C\right)^{T} P=-Q \\
P B=C^{T}
\end{gathered}
$$

and if $K_{e}=0$, then $\{A, B, C\}$ is said to be strictly positive real (SPR).

The model with the choice of the output $y \triangleq \theta=C_{0} \eta$ cannot be ASPR because the associated transfer function is of relative degree two. The ASPR condition can be satisfied if we make a judicious choice of the output variable of the form

$$
\begin{aligned}
y & =(\dot{\theta}+\mu \theta)=C_{0} \dot{\eta}+\mu C_{0} \eta \\
& =\left(\begin{array}{cc}
\mu C_{0} & C_{0}
\end{array}\right) x \triangleq C x
\end{aligned}
$$

where $\mu$ is positive real number.

Mufti $^{17}$ has shown that for a choice of

$$
\mu<\mu^{\star}=\min \left\{2 \zeta \omega_{i}, i=1, . ., 2 n\right\}
$$

there exists matrices $P$ and $Q$ which satisfy (21) for $K_{e}=0$ and therefore, the system is in fact, strictly positive real. This is also easily verified by computing the transfer function relating $y$ and $u$, which is

$$
\begin{gathered}
\frac{\hat{y}(s)}{\hat{u}(s)}=\sum_{i=1}^{2 n} \frac{c_{0 i} b_{1 i}(s+\mu)}{s^{2}+2 \zeta \omega_{i} s+\omega_{i}^{2}} \\
=\sum_{i=1}^{2 n} H_{i}(s)
\end{gathered}
$$

where $C_{0}=\left(c_{01}, \ldots, c_{0,2 n}\right)$ and $B_{1}=\left(b_{11}, \ldots, b_{1,2 n}\right)$. For the model under consideration, computing the matrices $C_{0}$ and $B_{1}$, one finds that

$$
c_{0 i} b_{1 i}>0, i=1, \ldots, 2 n
$$

and therefore, each of the transfer functions $H_{i}(s)$ with $\mu<2 \delta \Omega_{i}$ is SPR. Apparently, the parallel combination of SPR transfer functions is also SPR.

Later, even though $(A, B, C)$ is SPR, we shall introduce additional output feedback for modifying the transient characteristics. It may be noted that in fact, for any real number $K_{e}>0$, the system 
$\left\{\left(A-K_{e} B C\right), B, C\right\}$ remain SPR. This is easily verified by noting that SPR system $\{A, B, C\}$ implies that there exist $P>0$ and $Q>0$, such that

$$
\begin{aligned}
A^{T} P+P A & =-Q \\
P B & =C^{T}
\end{aligned}
$$

Subtracting both sides by $K P B C$ and $K_{e} C^{T} B^{T} P$ in (24) and noting that $P B=C^{T}$, one obtains

$$
P\left(A-K_{e} B C\right)+\left(A-K_{e} B C\right)^{T} P=-Q-2 K_{e} C^{T} C<0
$$

and therefore, same matrix $P$ solves (28). However, a good value of $K_{e}$ is not known. Therefore, we intend to design a controller which will adaptively seek a good output feedback gain $K_{e}$ for control.

Now the design of the controller for the asymptotic tracking of the reference fin angle $y_{m}$ is considered. When perfect tracking occurs, i.e, $y=y_{m}$ for $t \geq 0$, let $x^{\star}$, $\mathrm{u}^{\star}$ and $y^{\star}$, respectively denote the corresponding plant state, input and output trajectories of the ideal model (22) for perfect tracking satisfying

$$
\begin{array}{r}
\dot{x}^{\star}=A x^{\star}+B u^{\star} \\
y^{\star}=C x^{\star}=C_{m} x_{m}=y_{m}
\end{array}
$$

In the command generator tracker theory ${ }^{16,17}$, it is assumed that the starred signals satisfy

$$
\begin{aligned}
& x^{\star}=S_{11} x_{m}+S_{12} u_{m} \\
& u^{\star}=S_{21} x_{m}+S_{22} u_{m}
\end{aligned}
$$

where $S_{i j}$ are constant matrices. We assume here that $u_{m}$ is a step function. Indeed for the existence of matrices $S_{i j}$, one needs to satisfy only a mild (CGT) condition. For the solvability of (31), it is sufficient that, the transfer function $C(S I-A)^{-1} B$ has no zeros at the origin or in common with any eigenvalue of $A_{m}$. For the model under consideration, this CGT condition holds.

Defining $\tilde{x}=x^{\star}-x$, one obtains the state error equation

$$
\begin{gathered}
\dot{\tilde{x}}=A \tilde{x}+B\left(u^{\star}-u\right) \\
=\left(A-B K_{e}^{\star} C\right) \tilde{x}+B\left[K_{e}^{\star} \tilde{y}+S_{21} x_{m}+S_{22} u_{m}-u\right]
\end{gathered}
$$

where $\tilde{y}=y_{m}-y=C_{m} x_{m}-C x=C x^{\star}-C x=C \tilde{x}$ and $K_{e}^{\star}>0 . S_{21}, S_{22}$ are treated as unknown. The feedback gain $K_{e}^{\star}$ denotes an unknown gain which gives good perfomance.

Define $\nu=\left[\begin{array}{lll}K_{e}^{\star} & S_{21} & S_{22}\end{array}\right]^{T} \in \Re^{m+2}$. Then the error equation (32) can be written as

$$
\dot{\tilde{x}}=A_{a} \tilde{x}+B\left(\nu^{T} \Phi-u\right)
$$

where $\Phi=\left[\begin{array}{lll}\tilde{y} & x_{m}^{T} & u_{m}\end{array}\right] \in \Re^{m+2}$ and $A_{a}=\left(A-B K_{e}^{\star} C\right)$.

The control law is chosen as

$$
u=\hat{\nu}^{T} \Phi+\nu_{p}^{T} \Phi
$$

Here $\hat{\nu}$ denotes an estimate of the actual parameter vector $\nu$ and $\nu_{p}$ is a proportional gain.

Substituting the control law in (33) gives

$$
\dot{\tilde{x}}=A_{a} \tilde{x}+B\left[\tilde{\nu}^{T} \Phi-\nu_{p}^{T} \Phi\right]
$$

where $\tilde{\nu}=\nu-\hat{\nu}$ is the parameter estimation error.

For the derivation of the adaptation law, consider a quadratic Lyapunov function

$$
V=\tilde{x}^{T} P \tilde{x}+\tilde{\nu}^{T} \Gamma \tilde{\nu}
$$

where $\Gamma$ is a positive definite symmetric matrix (denoted as $\Gamma>0$ ). The derivative of $V$ along the solution of (22) is given by

$$
\dot{V}=\tilde{x}^{T}\left(P A_{a}+A_{a}^{T} P\right)+2 \tilde{x}^{T} P B\left[\tilde{\nu}^{T} \Phi-\nu_{p}^{T} \Phi\right]+2 \tilde{\nu}^{T} \Gamma \dot{\tilde{\nu}}
$$


Note that $\left\{A_{a}, B, C\right\}$ is SPR and $\tilde{x}^{T} P B=\tilde{x}^{T} C^{T}=\tilde{y}$. For making $\dot{V}$ negative, select the update law and gain $\nu_{p}$ of the form

$$
\begin{gathered}
\dot{\tilde{\nu}}=-\dot{\hat{\nu}}=\Gamma^{-1} \Phi \tilde{y} \\
\nu_{p}=\Gamma_{p} \Phi \tilde{y}
\end{gathered}
$$

where the matrix $\Gamma_{p} \geq 0$.

Substituting the adaptation laws (38) and (39) in (37) and using (28), one obtains

$$
\dot{V} \leq-\tilde{x}^{T} Q \tilde{x}-2 \lambda_{\min }\left(\Gamma_{p}\right)\|\Phi\|^{2} \tilde{y}^{2} \leq 0
$$

where $\lambda_{\min }\left(\Gamma_{p}\right)$ is the smallest eigenvalue of $\Gamma_{p}$. In view of (40), $\dot{V}$ is negative semidefinite. According to Lyapunov stability results ${ }^{22,24}$, it follows that $\tilde{x}(t)$ tends to zero as $t \rightarrow \infty$ and therefore, the target pitch angle is attained.

It is well known that the presence of disturbance input $d$ can cause parameter divergence. For obtaining robust stability, one modifies the update law as

$$
\dot{\hat{\nu}}=0
$$

when the error is small. With this dead-zone modification, one can show that all the signals and the tracking error are bounded in the closed loop system if $d \neq 0^{26}$.

\section{Simulation Results}

This section presents the simulation results for the smart fin (fin-beam model) including the model reference adaptive control law. The mechanical properties of the simulated model are taken from Yim et al. ${ }^{13}$. These are: $\rho_{b}=8,300\left(\mathrm{~kg} / \mathrm{m}^{3}\right), \rho_{p}=7,500\left(\mathrm{~kg} / \mathrm{m}^{3}\right), E_{b}=70 G P a, E_{p}=63 G P a$, $L=140 \mathrm{~mm}, b=25 \mathrm{~mm}, h_{b}=0.5 \mathrm{~mm}, h_{p}=0.127 \mathrm{~mm}, d_{31}=1.8 \mathrm{E}-10(\mathrm{~m} / \mathrm{volt})$. Using finite element method (with $\mathrm{n}=5$ elements), a state-variable representation of the fin-beam model of dimension 20 is obtained for simulation. The aerodynamic moment (14) is chosen for different angles of attack of the projectile based on the CFD analysis. By a linear approximation of the data obtained by the CFD analysis, the parameters of the aerodynamic moment are found to be $m_{a 0}=-0.005, p_{a}=+0.0263$ for $\alpha=-10^{\circ}$, and $m_{a 0}=0.005, p_{a}=-0.0286$ for $\alpha=+10^{\circ}$. The value of $\Gamma=5000000\left(I_{2 n \times 2 n}\right)$ and $\Gamma_{p}$ is chosen to be zero and therefore $\nu_{p}$ becomes zero. The minimum value of $\mu$ obtained is 0.5 . The initial values of $\hat{\nu}$ are taken as $[-10,0,0]^{T}$. The damping coefficient is taken as $\zeta=0.005$. The aerodynamic moment is chosen as

$$
m_{a}=a(\alpha)+b(\alpha) \theta
$$

where $\alpha$ is the angle of attack and $\theta$ is the fin angle. Simulation results are shown for different fin angle commands. The reference command trajectory is obtained using a first order command generator, with values $A_{m}=-6, B_{m}=6, C_{m}=1$.

\section{A. Adaptive Control: Fin Angle $=10^{\circ}$, Angle of attack $=-10^{0},+10^{0}$}

Figure. 2 and Figure. 3 show the simulation results for fin angle of $10^{\circ}$, with angles of attack $-10^{\circ}$ and $+10^{\circ}$. It is observed that the fin angle asymptotically converge to the desired value less than 6 seconds. The control input needed to deflect the fin to an angle of $10^{\circ}$ with angle of attack $-10^{\circ}$ is around 1800 volts while that for $+10^{\circ}$ is just above 2000 volts. We observe that for larger fin angle command the control input needed is much larger. It is also interesting to see that there is no overshoot for the flexible modes and they reach their equilibrium values at the steady state, in both the cases.

\section{Conclusion}

This paper considered the control of rotation angle of a smart projectile fin. A flexible beam with a piezoelectric active layer was used for rotating the fin. The model of the fin-beam system includes the aerodynamic moment which is a function of angle of attack of the projectile. A state variable model using finite element method was obtained. A model reference adaptive controller, based on the command generator tracker concept is designed. Simulation results show that the 
designed adaptive control system accomplishes precise fin angle control in spite of uncertainties in the fin-beam parameters and the aerodynamic moment coefficients.

\section{Acknowledgments}

This work is supported by the US Army Research Lab under Cooperative Agreement No. DAAD19-03-2-0007.

\section{References}

1 Batra, R.C., and Ghosh, K., "Deflection Control during Dynamic Deformations of a Rectangular Plate Using Piezoceramic Elements," AIAA Journal, Vol. 33, No. 8: Technical Notes, 1995, pp 1547-1548.

2 Lin, C.C., Hsu, C.Y., and Huang, H.N., "Finite element analysis on deflection control of plates with piezoelectric actuator," Composite Structure, Vol. 35, 1997, pp.423-433.

3 Rabinovitch, O., and Vinson, J., "On the design of piezoelectric smart fins for flight vehicles," Smart Materials and Structures, Vol. 12, 2003, pp 686-695.

4 Rabinovitch, O., and Vinson, J., "Smart fins: Analytical modeling and basic design concepts," Mech. Adv. Mater. Struct, Vol. 10, 2003, pp. 249-269.

5 Barrett,R., "Active plate and missile wing development using directionally attached piezoelectric elements," AIAA Journal, Vol. 32, No. 3, 1994, pp. 601-609.

${ }^{6}$ Bent, A.A., Hagood, N.W., and Rodgers, J.P., "Anisotropic actuation with piezoelectric fiber composites," Journal of Intelligent Materials Systems and Structures, Vol. 6, No.3, 1995, pp.338-349.

7 Shen, Y., and Homaifar, A., "Vibration Control of Flexible Structures with PZT Sensors and Actuators," Journal of Vibration and Control, 7, 2001, pp. 417-451.

8 Bailey, T. and Hubbard, J. E., "Distributed Piezoelectric-Polymer Active Vibration Control of a Cantilever Beam," J. of Guidance, Control, and Dynamics, Vol. 8 No. 5, pp. 605-611.

${ }^{9}$ Choi, S.B., and Lee,C.H., "Force Tracking Control of a Flexible Gripper Driven by Piezoceramic Actuator," ASME Trans. J. of Dynamic Systems, Measurement, and Control, Vol. 119, pp. 439-445.

${ }^{10}$ Baz,A., and Poh, S., "Performance of an active control system with piezoelectric actuator," Journal of Sounds and Vibration, Vol. 126, No 2, 1988, pp 327-343.

11 Choi, S.B., Cheong, C.-C., and Lee,C.-H., "Position tracking control of a smart flexible structure featuring a piezofilm actuator," Journal of Guidance, Control and Dynamics, Vol. 19, No 6, Nov-Dec, 1996.

12 Yim, W., and Singh, S.N., "Variable structure adaptive control of a cantilever beam using piezoelectric actuator," Journal of Vibration and Control, Vol. 6, 2000, pp 1029-1043.

13 Yim, W., Singh, S.N., and Trabia, M., "Adaptive Control of Projectile Fin Angle Using Piezoelectric Beam Actuator," Proceedings of SPIE Smart Structures and Materials, March, 2004.

14 Trabia, M., Yim, W., "Fuzzy Logic Control of Projectile Fin Angle Using Piezoelectric Actuator," IMECE 2004.

15 Hyland, D. C., Junkins, J. L., and Longman, R. W., "Active Control Technology for Space Structures," Journal of guidance, Control and Dynamics, 16, 1993, pp 801-821.

16 Kaufmann, H., Barkana, I., Sobel, K., "Direct Adaptive Control Algorithms, "Springer-Verlag, New York, 1998.

17 Mufti, I., H., "Model Reference Adaptive Control for large structural systems," AIAA Journal of Guidance, Control and Dynamics, 1987, pp 507-509.

18 Annaswamy, A. M., and Clancy, D. J., "Adaptive Control Strategies for Flexible Space Structures," IEEE Transactions on Aerospace and Electronic Systems, Vol. 32, No. 3, July 1996.

19 Kelkar, A., and Joshi, S., "Control of Nonlinear Multibody Flexible Space Structures, " Springer, NewYork, 1996.

20 Singh, S. N., Zhang,R., "Adaptive Output Feedback Control of Spacecraft with Flexible Appendages by Modeling Error Compensation," Acta Astronautica 54, 2004, pp. 229-243.

21 Singh, S, N., and Araujo, A. D. de., "Adaptive Control and Stabilization of Elastic Spacecraft," IEEE Transactions on Aerospace and Electronic Systems, Vol. 35, January 1999, pp. 115-122.

22 Singh, S. N., "Robust Nonlinear Attitude Control of Flexible Spacecraft, " IEEE Transactions on Aerospace and Electronic Systems, Vol. AES-23, No. 3, May, 1987, pp. 380-387. 
${ }^{23}$ Mani, S., Singh, S. N., Parimi, S. K., Yim. W., Trabia, M., "Adaptive Rotation of a Smart Projectile Fin by Piezoelectric Flexible Beam Actuator, " To appear in Journal of Vibration and Control, 2005.

24 Vidyasagar, M., "Nonlinear Systems Analysis," SIAM Publications, NJ, 2002.

${ }^{25}$ Fradkov, A. L., Miroshnik, I. V., Nikiforov, V. O., "Nonlinear and Adaptive Control of Complex Systems, " Kluwer Academic Publishers, 1999.

26 Narendra, K. S., Annaswamy, A. M., "Stable Adaptive Systems, " Prentice-Hall, NJ, 1989.

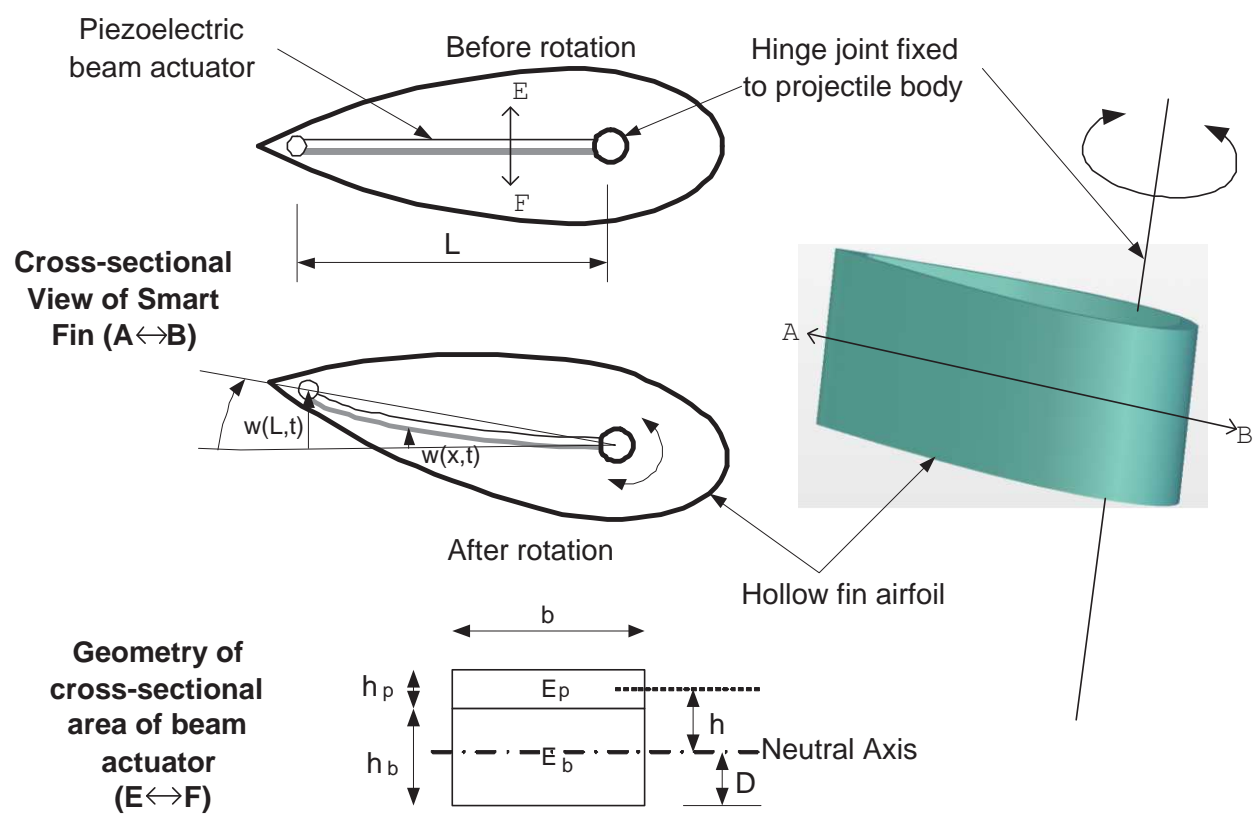

Figure 1. Configuration of Smart Fin 

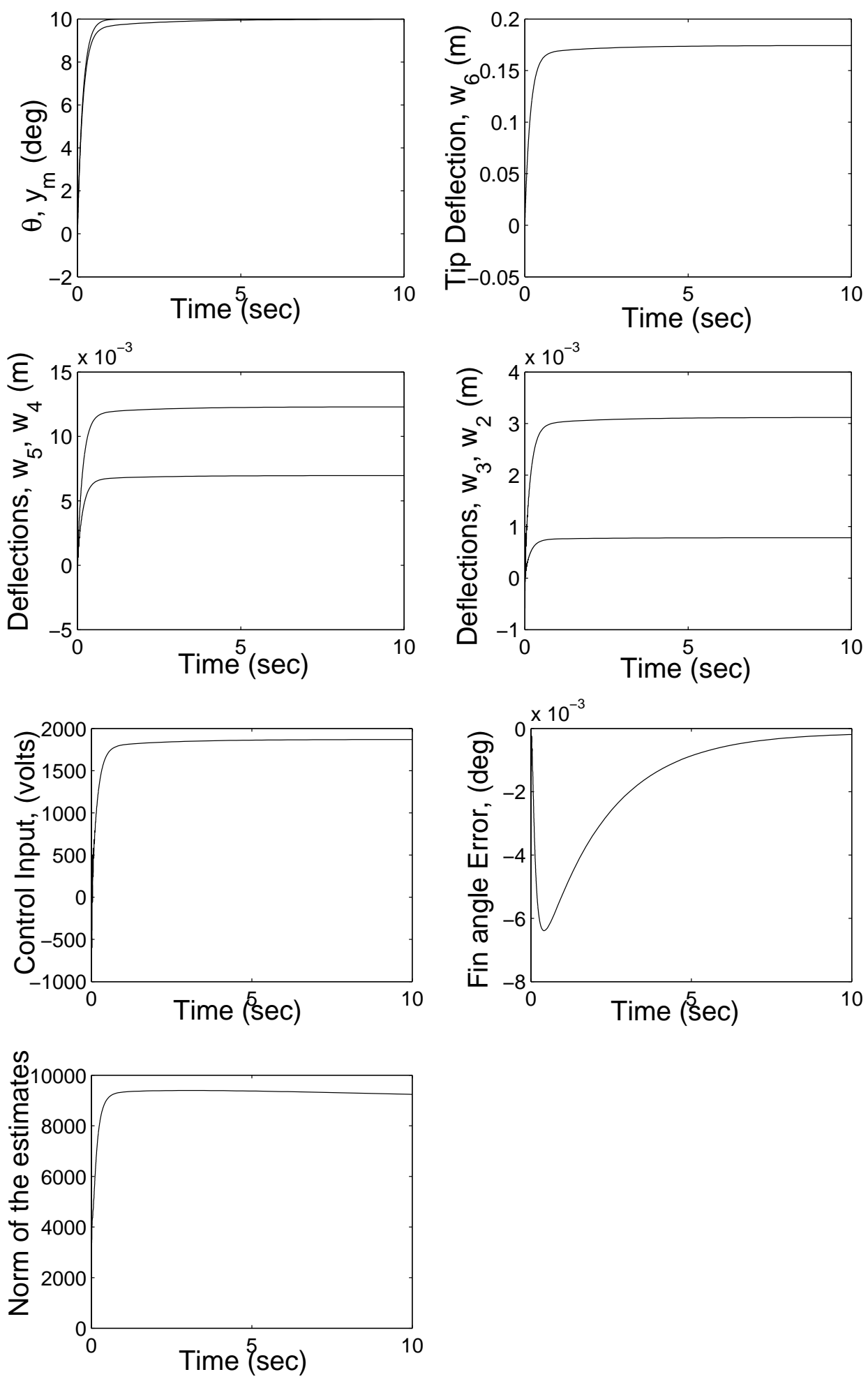

Figure 2. Adaptive Control: Fin Angle $=10^{0}$, Angle of attack $=-10^{0}$ 

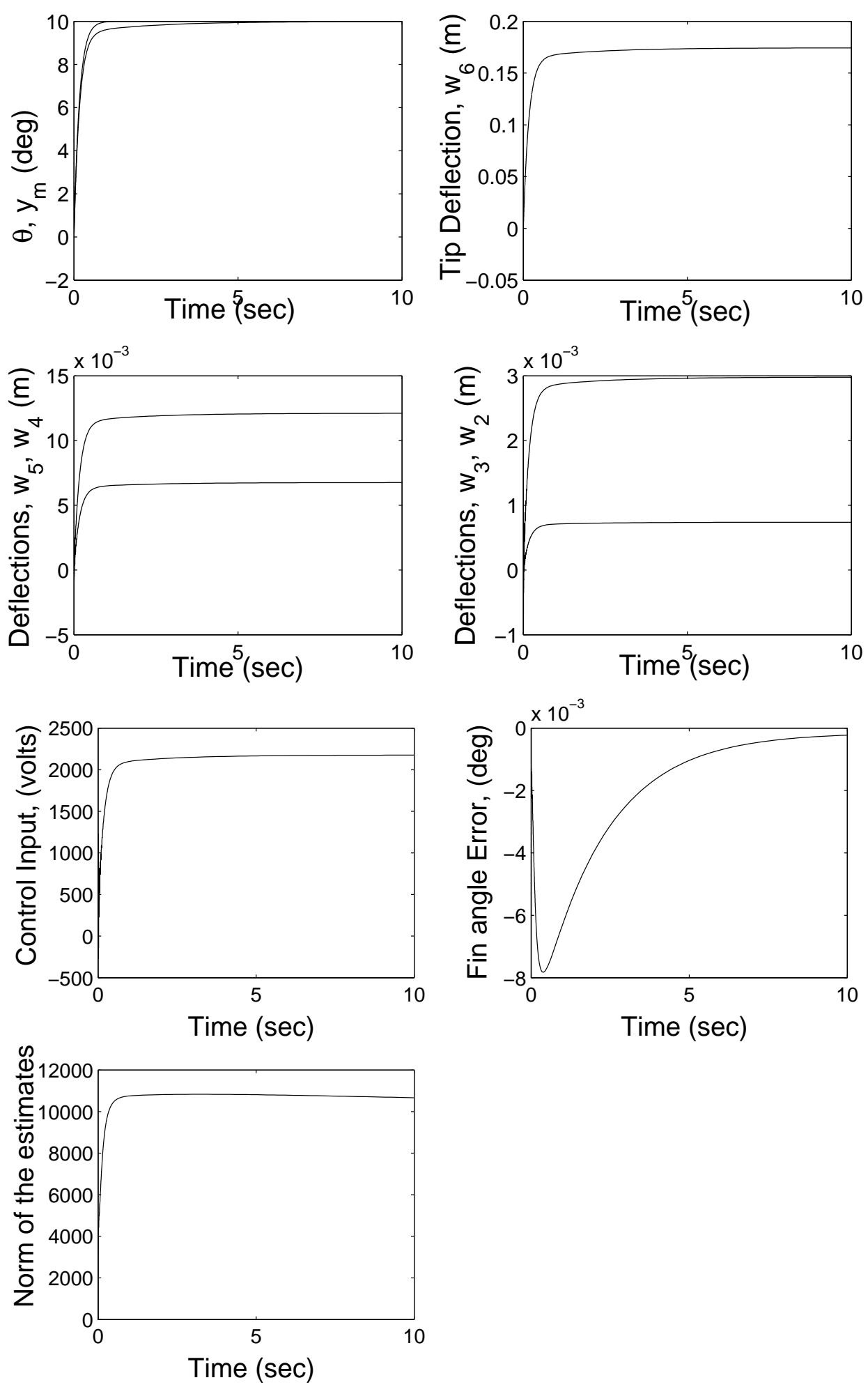

Figure 3. Adaptive Control: Fin Angle $=10^{0}$, Angle of attack $=10^{0}$ 